4027 and 7741. Also we need not consider a closed-circuit circulation pattern, as interstellar matter will be removed by star formation and presumably replenished by injection from the corona.

Shobbrook: Although there appears to be definite evidence for leading of these small arms, I believe I am correct in saying that there are no positive data indicating a leading motion of the large main arms in any of the spirals or irregulars.

de Vaucouleurs: The main arms of these magellanic irregulars are trailing, but if one calls the small, curved parts "arms", then one of them at least is definitely leading.

Feast: Do you believe that your model of the LMC is consistent with the observed rotation curve of gas and stars? There appears to be no evidence in these results of streaming along the bar.

de Vaucouleurs: There is no evidence because no one has yet analysed the velocity data with this possibility in mind. Radio astronomers, using high resolution, should look for gas streaming along the bar of the Large Cloud.

Kerr: There is no evidence in the radio observations so far for a streaming motion along the bar, but such a motion would be quite difficult to distinguish from error in the assumed galactic rotation velocity.

de Vaucouleurs: If we assume a streaming motion along the bar of $\pm 50 \mathrm{~km} / \mathrm{sec}$, the line-ofsight component should be only about \pm 15 to $20 \mathrm{~km} / \mathrm{sec}$, which certainly would be difficult to disentangle from other motions.

\title{
59. COMPARISON OF NEUTRAL HYDROGEN IN NGC 55 AND THE LMC
}

\section{B. J. Robinson and K. J. van Damme CSIRO Radiophysics Laboratory, Sydney}

Twenty-one-cm neutral hydrogen emission from NGC 55 has been measured with the 210-foot radio telescope at Parkes and a low-noise receiver (Robinson 1963). The beamwidth at $21 \mathrm{~cm}$ is 13.5. NGC 55 has been classified by de Vaucouleurs as a SB(s)m system similar to the LMC, seen edgewise and with the luminous bar nearly along the line of sight (de Vaucouleurs 1961). The outer isophote published by de Vaucouleurs has dimensions of 45 by $9^{\prime}$, centred about 2.'5 to the south-following side of the bright "nucleus" in the centre of the bar.

Hydrogen-line profiles measured at $8^{\prime}$ intervals along the major axis are shown in Figure 1. The displacement of the velocity of the profile peak clearly reveals the rotation of the system. The velocities of the peaks (relative to the Sun) have been plotted in Figure 2. The velocities agree moderately well with those determined for three emission objects by de Vaucouleurs (1961). The rotation curve shows a marked asymmetry about the centre of the bar, similar to that found previously for the LMC (Kerr and de Vaucouleurs 1955). The rotation in NGC 55 is symmetrical about a point on the major axis displaced $3^{\prime}$ on the south-following side of the "nucleus". Assuming a corrected distance modulus of $26 \cdot 3$ for NGC 55 (de Vaucouleurs 1961), the rotation centre lies $1.5 \mathrm{kpc}$ from the centre of the bar. The corresponding distance in the LMC is 800 pc.

The mass contained within a radius of $30^{\prime}$ from the centre of rotation is $2 \times 10^{10} M_{\odot}$, giving $M / L \simeq 5 \cdot 1$. The total mass will be somewhat greater than this. There will be a small contribution (perhaps 10\%) from matter lying more than 
$30^{\prime}$ from the centre. Also the rotation curve of Figure 2 will be flatter than the true rotation curve because of the limited resolution and because the measured HI emission is an average along the line of sight.
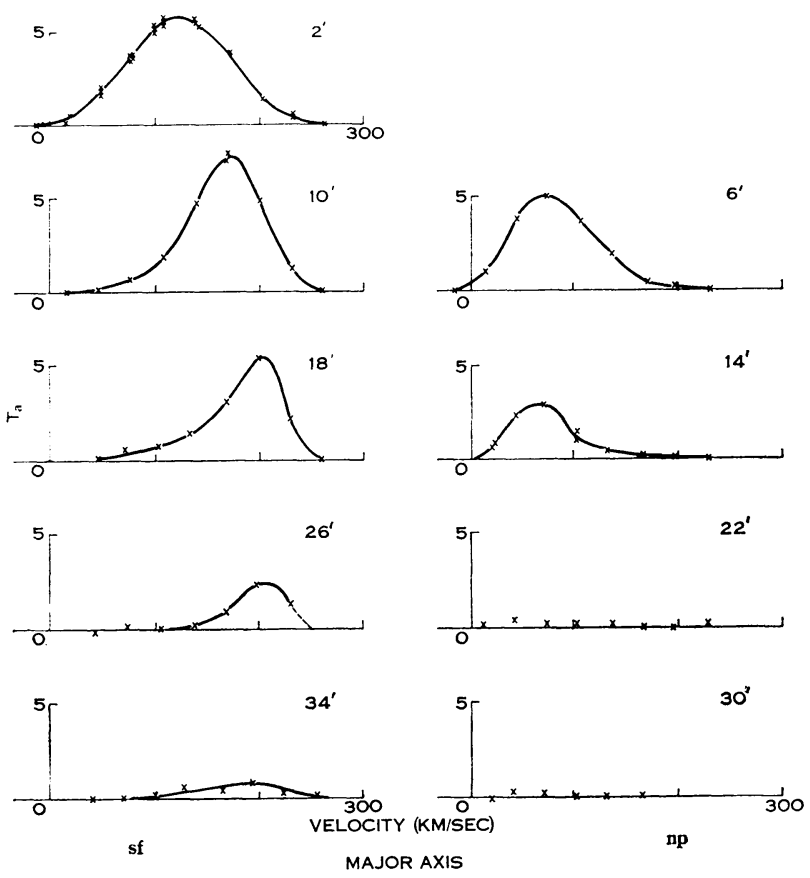

Fig. 1. $-21-\mathrm{cm}$ line profiles at points on the major axis of NGC 55. The number against each profile is the distance (in minutes) from the "nucleus" in the centre of the bar.

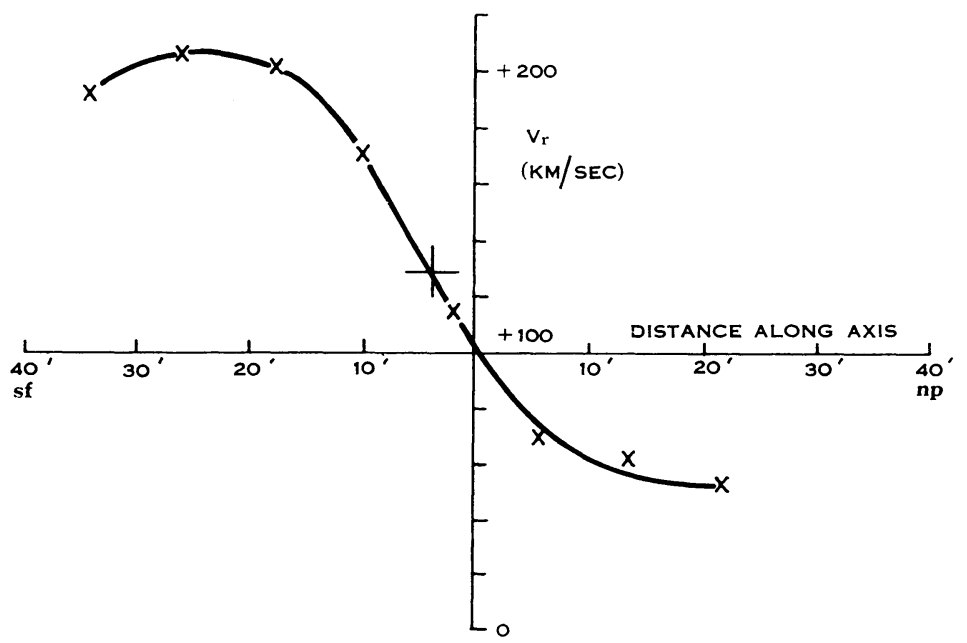

Fig. 2.-Rotation curve for NGC 55. Distances are measured from the nucleus. 
Integration of the area under the profiles of Figure 1 shows that there is about $50 \%$ more hydrogen on the following side of the nucleus than preceding it. The centroid of the HI distribution is displaced 2.5 from the nucleus, corresponding to a distance of $1.3 \mathrm{kpc}$. In the LMC the HI distribution is similarly displaced, the centroid being about $1 \cdot 2 \mathrm{kpc}$ from the centre of the bar. Measurements along the minor axis of NGC 55 also show a slightly asymmetrical HI distribution. More hydrogen is found in the sf quadrant (referred to the major axis) than in the other quadrants. Integration of all scans across the system yields a hydrogen mass of $2 \cdot 2 \times 10^{9}$ solar masses, approximately three times that for the LMC. The ratio $M_{\mathrm{H}} / M_{\mathrm{T}}$ is thus $0 \cdot 11$, the ratio $M_{\mathrm{H}} / L \simeq 0 \cdot 55$ (in solar units).

These measurements provide support for de Vaucouleurs' morphological comparison of NGC 55 with the LMC. In both systems the centre of symmetry of the rotation curve, the centroid of the $\mathrm{HI}$ distribution, and the centroid of the luminosity distribution are displaced from the bar. NGC 55 is about three times as massive and has a similar percentage of neutral hydrogen.

\title{
References
}

Kerr, F. J., and de VAucoulevrs, G. (1955).-Aust. J. Phys. 8: 508-22.

Robixson, B. J. (1963).-Proc. I.R.E. (Aust.) $24: 119-27$.

de Vaucoulecrs, G. (1961).-Ap. J. $133: 405-12$.

\section{Discussion}

de Vaucouleurs: You should not expect a large broadening of the $21-\mathrm{cm}$ line profile at the position of the bar because the beamwidth covers a large area and only a very small fraction of the $\mathrm{HI}$ in the beam will have the maximum stream velocity of $\pm 50 \mathrm{~km} / \mathrm{sec}$ or so.

Robinson: If only a small fraction of the total hydrogen near the bar is streaming outwards, it would be difficult to detect with a beamwidth of $14^{\prime}$.

Aller: NGC 55 contains a bright nebulosity comparable perhaps with 30 Doradus. D. J. Faulkner and $\mathrm{I}$ observed strong lines of [OIII] and of $\mathrm{H}$ indicating a level of excitation greater than that of many diffuse galactic (or LMC) emission nebulae.

\section{REGIONS HII DANS LES NUAGES DE MAGELLAN ET LES GALAXIES PROCHES}

\author{
G. Courtes
}

Observatoire de Marseille

\section{Nuages de Magellan}

Les clichés du Grand et du Petit Nuage décrits dans cet article ont été pris à travers un filtre interférentiel d'une qualité analogue aux précédents (voir dans le même Symposium: "Distribution des vitesses radiales des régions HII dans la direction du Centre Galactique"), mais centrés évidemment sur la longueur d'onde correspondante au déplacement Doppler-Fizeau dû à la vitesse radiale des Nuages qui, comme on le sait, est de l'ordre de $+200 \mathrm{~km} / \mathrm{sec}$. La largeur de la bande passante était de $10 \AA$. 